\title{
Electron diffraction studies of supersonic jets. III. Clusters of $n$-butane
}

\author{
Richard K. Heenan and Lawrence S. Bartell \\ Department of Chemistry, University of Michigan, Ann Arbor, Michigan 48109 \\ (Received 23 September 1982; accepted 19 October 1982)
}

\begin{abstract}
Expansions of $n$-butane from small nozzles were observed to generate large clusters, provided that enough helium or neon carrier gas accompanied the butane to remove the heat of condensation rapidly and to produce low temperatures. Although expansions of neat $n$-butane led to substantial conformational cooling under some circumstances, clusters were never seen without carrier gas. Both thin-plate and tapered glass nozzles yielded clusters. Diffraction patterns of the clusters are characteristic of a liquidlike structure perhaps as cold as $100 \mathrm{~K}$. No evidence for inclusion of carrier gas atoms in the clusters was found. Cluster scattering signals are stronger and show less noise than those which could be expected from bulk liquid by $\mathrm{x}$-ray or neutron diffraction. Their interference terms are dominated by nearest neighbor intermolecular $\mathbf{C . . . H}$ contacts. Information about the distribution of these contacts has not yet been deduced in computer simulations of liquid butane but should soon become available for comparison.
\end{abstract}

\section{INTRODUCTION}

Despite the popularity of the $n$-butane molecule for calculations on the dynamics of liquids, ${ }^{1-5}$ comparatively little experimental structural information exists for $n$ butane, other than for the gas phase. Following our successful observation by electron diffraction of amorphous benzene clusters in a seeded molecular beam, ${ }^{6}$ we decided to investigate the cooling of $n$-butane in similar supersonic expansions of helium or neon. If microdroplets of cold liquid penetrable by electrons could be produced, as with benzene, this might help elucidate the liquild structure of $n$-butane, for which at least one $x$-ray diffraction investigation is in progress. ${ }^{7}$ Since benzene molecules were unable to organize onto a regular crystalline lattice on the time scale of our experiment, butane molecules would be even less likely to do so given their irregular shape, conformational degrees of freedom, and much lower freezing point $(134.6 \mathrm{~K}$, as compared with $267.5 \mathrm{~K}$ for benzene). Since structure determinations of neither liquid nor solid, bulk $n$-butane are yet available, there is little information with which to compare our cluster diffraction patterns. Infrared and Raman spectra have shown three distinct forms for crystalline $n$-butane, each involving only trans conformers. ${ }^{8}$ At very low temperatures a triclinic form exists which has a reversible transition at $110 \mathrm{~K}$ to a disordered structure. Rapid cooling of the disordered structure produces a metastable monoclinic form. A lattice packing calculation for $n$-butane suggested that a structure related to that for $n$-hexane, but with nonparallel chain axes, might present the lowest energy. ${ }^{8}$

There seems to be no direct experimental evidence on the conformational ratio in liquid $n$-butane. Recent molecular dynamics ${ }^{4}$ and Monte Carlo ${ }^{1-3}$ simulations suggest that at normal densities there is no significant conformational change between gas and liquid phases in the vlcinity of the boiling point. However, spectroscopic inferences of the conformational $\Delta H^{\circ}$ in the liquid yield values significantly lower $\left[2.26(42),{ }^{9} 2.33(5),{ }^{10}\right.$ and $\left.2.39(46)^{11} \mathrm{~kJ} \mathrm{~mol}^{-1}\right]$ than that for the gas phase $\mathrm{s}^{12}[3.72(12)$ $\mathrm{kJ} \mathrm{mol}^{-1}$. At least at low temperatures this would suggest an increased preference for the gauche conforma- tion in the liquid compared to the gas at the same temperature. Although optimization of packing forces in condensed phases might be expected to lead to some conformational preference, available evidence warns against a glib interpretation. On the one hand, both Raman experiments and computer simulations ${ }^{2}$ indicate for the liquid that increasing pressure, which should make packing optimization more important, leads to increased gauche concentration. On the other hand, the solid is reported to be all trans even though gas phase thermodynamic parameter $\mathrm{s}^{12}$ predict about $7 \%$ gauche at the freezing point.

\section{EXPERIMENTAL}

Due to the large number of experimental variables that we wished to investigate, only pairs of diffraction patterns were taken under identical conditions in most cases. Details for selected plates are given in Table I. Plates with numbers less than 100 , all with thin plate nozzles and $R^{3}$ rotating sector, were produced one year earlier than the rest and used Kodak electron image emulsions. The remainder of the patterns were recorded on Kodak medium projector slide plates ( 4 in $\times 5 \mathrm{in})$. The camera height was $214 \mathrm{~mm}$ in all cases. Gas mixtures were made by passing monatomic carrier gas over liquid $n$-butane contained in a brass chamber which was most often suspended in a cold bath at $0^{\circ} \mathrm{C}$. Butane and helium mixtures for plates 466-74 and 47784 were, however, accurately premixed to $66 \%$ and $85 \%$ butane, respectively. Gases were obtained from Air Products Ltd., helium (99.995\%), neon (99.99\%), and $n$-butane (99.5\%). All were used directly from their cylinders, without further purification. Previous papers in this series describe the apparatus and data reduction methods in detail. 6,13 Intensities for the plates illustrated in this work are available as supplemental material. ${ }^{14}$

\section{MOLECULAR MODEL}

A molecular description was adopted with five geometric parameters to characterize the gauche and trans mixture. It was similar to that formulated by Bradford, 
TABLE I. Gas expansion conditions and residual cluster signals for $n$-butane diffraction patterns. ${ }^{2}$

\begin{tabular}{|c|c|c|c|c|c|c|c|c|}
\hline Nozzle & $\begin{array}{l}\text { Diameter } \\
\text { (mm) }\end{array}$ & $\begin{array}{l}\text { Length } \\
\text { (mm) }\end{array}$ & $\begin{array}{l}\text { Plate } \\
\text { nos. }\end{array}$ & $\begin{array}{l}\text { Relative } \\
\text { exposure }^{b} \\
\text { (s) }\end{array}$ & $\begin{array}{l}\text { Total } \\
\text { pressure } \\
\text { (psia) }\end{array}$ & $\begin{array}{l}n \text {-butane } \\
\text { pressure } \\
\text { (psia) }\end{array}$ & $\begin{array}{l}\text { Height } \\
\text { of } \\
\text { residual }^{c}\end{array}$ & $\begin{array}{l}\text { Rotating } \\
\text { sector } \\
\text { exponent }\end{array}$ \\
\hline \multirow[t]{9}{*}{ Thin plate } & 0.01 & $\cdots$ & $257 / 8$ & 5 & Pure & 25 & 0.0 & 3 \\
\hline & & & $263 / 4$ & 2 & Pure & 25 & 0.0 & 2 \\
\hline & & & 52 & 10 & Pure & 25 & 0.0 & 3 \\
\hline & & & $47 / 8$ & 9 & $70 \mathrm{He}$ & 15 & 0.24 & 3 \\
\hline & & & 53 & 23 & $70 \mathrm{He}$ & 6.5 & 0.16 & 3 \\
\hline & & & 55 & 28 & $35 \mathrm{He}$ & 6.5 & 0.16 & 3 \\
\hline & 0.05 & $\cdots$ & $63 / 4$ & 37 & $110 \mathrm{He}$ & 8.6 & 0.22 & 3 \\
\hline & 0.025 & $\cdots$ & $77 / 8$ & 33 & Pure & 37 & 0.0 & 3 \\
\hline & & & $71 / 2$ & 32 & $440 \mathrm{He}$ & $\sim 21$ & 0.28 & 3 \\
\hline \multirow[t]{2}{*}{ Tubular } & 0.12 & 5.6 & $267 / 8$ & 5 & $70 \mathrm{He}$ & 15 & $\sim 0.06$ & 2 \\
\hline & & & $269 / 70$ & 4 & $100 \mathrm{He}$ & 15 & 0.13 & 2 \\
\hline \multirow[t]{3}{*}{ Glass 1} & 0.145 & 20 & $312 / 3$ & 2 & Pure & 17 & 0.0 & 2 \\
\hline & & & $314 / 5$ & 2 & $30 \mathrm{He}$ & 17 & 0.16 & 2 \\
\hline & & & 322 & 0.4 & $50 \mathrm{He}$ & 15 & 0.16 & 2 \\
\hline \multirow[t]{3}{*}{ Glass 3} & 0.275 & 20 & $332 / 3$ & 0.5 & Pure & 25 & 0.0 & 2 \\
\hline & & & $334 / 5$ & 0.4 & Pure & 15 & 0.0 & 2 \\
\hline & & & $337 / 8$ & 0.3 & $30 \mathrm{He}$ & $\sim 15$ & 0.04 & 2 \\
\hline \multirow[t]{2}{*}{ Glass 1} & 0.145 & 20 & $362 / 3$ & 0.4 & $60 \mathrm{Ne}$ & 15 & 0.17 & 2 \\
\hline & & & $364 / 5$ & 1.7 & $20 \mathrm{Ne}$ & 15 & 0.14 & 2 \\
\hline \multirow[t]{2}{*}{ Glass 3} & 0.275 & 20 & $367 / 8$ & 0.5 & $20 \mathrm{Ne}$ & 15 & 0.0 & 2 \\
\hline & & & $369 / 70$ & 0.4 & $20 \mathrm{Ne}$ & $\sim 5$ & 0.26 & 2 \\
\hline Glass 8 & 0.296 & 25 & 402 & 0.3 & $20 \mathrm{He}$ & 15 & 0.0 & 2 \\
\hline \multirow[t]{4}{*}{ Glass 9} & 0.240 & 30 & $466-469$ & 0.5 & $30 \mathrm{He}$ & 20 & 0.0 & 3 \\
\hline & & & $471-474$ & 0.5 & $27 \mathrm{He}$ & 18 & 0.0 & 2 \\
\hline & & & $477-479$ & 0.7 & $25 \mathrm{He}$ & 21.3 & 0.0 & 2 \\
\hline & & & $481-484$ & 0.6 & $25 \mathrm{He}$ & 21.3 & 0.0 & 3 \\
\hline
\end{tabular}

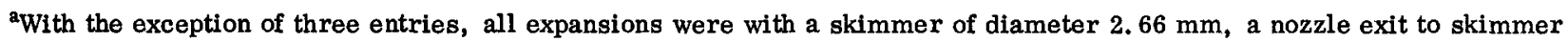
entrance distance of $8 \mathrm{~mm}$, and a nozzle exit to electron beam distance of 13-15 mm. Nozzle-skimmer distances were 13 $\mathrm{mm}$ for 53 and 55 , and $5 \mathrm{~mm}$ for $77 / 8$. $14.7 \mathrm{psia}=1 \mathrm{~atm}=760$ Torr.

${ }^{b}$ Relative exposure is the approximate time for unit peak optical density on the photographic plate using our $R^{2}$ rotating sector and an electron beam current of $10^{-6} \AA$. Practical exposures for our $R^{3}$ sector would be 14 times longer than indicated here.

${ }^{c}$ Residual height is a very approximate measure of the amount of intermolecular cluster scattering and is the height in sM(s) of the cluster signal around $s=5 \AA^{-1}$ using a $100 \%$ trans monomer model.

Fitzwater, and Bartell (BFB) in a previous electron diffraction study of $n$-butane. ${ }^{15}$ In most of our present investigations data covered only a limited range of scattering variable $s$. This, coupled with often large underlying cluster scattering precluded a complete determination of structural parameters. Vibrational amplitudes and shrinkage were calculated by BFB from a spectroscopic force field. More recent spectroscopic data would probably not significantly alter their results. One difference between the BFB model and that adopted here is that our Morse asymmetry parameters were set to $2.0 \AA^{-1}$ only for bonded and 1,3 distances, not for all internuclear distances. Leveled intensities were weighted proportional to $s$ in structural least-squares analyses. Electron scattering factors were taken from Sellers et $a l_{\circ}{ }^{16}$ Conformational ratios were determined directly by least squares rather than by BFB's method of searching for a best fit by repeated calculations with fixed mole fractions. Reference structural parameters were determined for pure $n$-butane, as described in the following paper, ${ }^{17}$ to be used to calculate monomer scat- tering in all the analyses here. Due to the configuration of the supersonic jet we do not have a unique, sharply defined camera height. Accurate determination of interatomic distances requires calibration against a known molecular structure. In this work where we were more interested in qualitative effects, small variations in camera height from run to run were absorbed in the analysis by allowing the $\mathrm{C}-\mathrm{C}$ bond length to refine as needed to give a good fit to $n$-butane monomer intensities. Some additional flexibility in the monomer intensity was included by varying the mean CCC angle, though this changed little (always being within $114 \pm 0.5^{\circ}$ ). Also varied were a smooth background function and helium or neon carrier gas subtractions, as appropriate. ${ }^{19}$ Mass fractionation of heavier species towards the center of the gas jet makes it impossible to predict with precision the proportion of carrier gas scattering, even though the composition of the reservoir mixture is known. All the illustrations of experimental cluster intensities given here were obtained after subtraction of first a "systematic residual" and then an adjusted $n$-butane 
monomer intensity from the total molecular scattering. Pure butane plates $263 / 4$, fit with the reference structure given in the following paper, were used to provide the systematic residual. This is an empirical correction to allow for deficiencies in the standard electron scattering expressions applied in our theoretical $M(s)$ curves. The effectiveness of such a correction when looking for small changes in scattering has been confirmed for $\mathrm{SF}_{6}{ }^{18}$ In the present case the residual correction, shown in Fig. 2, improved not only the fit of patterns of monomeric butane but also the agreement in shape between cluster intensities of different sizes.

\section{INFERENCE OF CLUSTER STRUCTURE}

In prior studies of liquids and amorphous materials by diffraction techniques it has been customary to interpret results in terms of radial distribution functions derived from scattered intensities. We have chosen not to do this in the current work for two reasons. First, the intensity data presently available are cut off inside $s \approx 3 \AA^{-1}$ by the rotating sector employed to make quantitative intensity measurements possible. Accounting for the missing intensities would introduce substantial uncertainties in the radial distribution functions. Second, our long experience in analyzing gas-phase diffraction patterns has shown the advantages of directly comparing observed and calculated intensities rather than radial distribution functions. For the present preliminary studies it will suffice to make qualitative assessments of the plain cluster signals that arise when microdroplets are formed. In the future, when sectors currently under construction will increase the data range covered and when more extensive computer simulations of liquid structures and corresponding intensities will be available, it will become feasible to analyze structures more quantitatively.

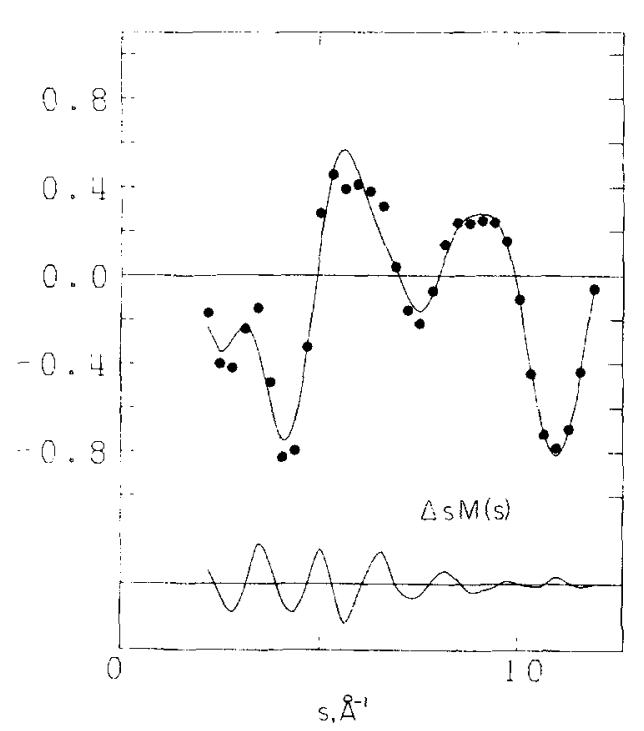

FIG. 1. Electron diffraction reduced intensities $\operatorname{SM}(s)$ for $n-$ butane ( $\sim 5$ psia) in neon at a reservoir pressure of 20 psia, through a tapered glass nozzle of $0.275 \mathrm{~mm}$ entrance diameter (plate 369 of Table D. In upper trace, points are experimental data, solid line is for $n$-butane monomer. Lower trace is difference, representing intermolecular cluster scattering.

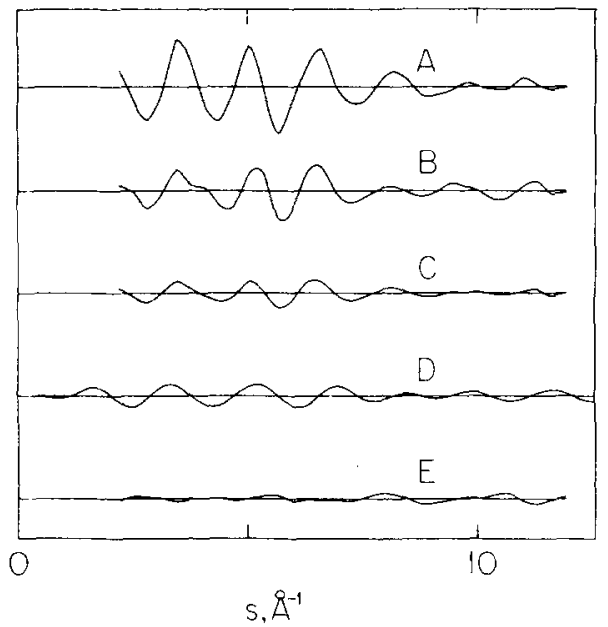

FIG. 2. Reduced intensities $\mathrm{sM}(s)$. (a) Cluster scattering for plate 369, as in Fig. 1. (b) Cluster scattering for plates $314 / 5, n$-butane in helium, subtraction assuming $92 \%$ trans monomer scattering (see Table III of Ref. 17). (c) Cluster from plates $364 / 5, n$-butane in neon, $88 \%$ trans monomer scattering. (d) Theoretical contribution of conformational cooling from $68 \%$ trans (room temperature equilibrium) to $100 \%$ trans (retaining room temperature amplitudes of vibration). (e) Systematic error, residual from pure butane plates $263 / 4$, see the text and Ref. 17. Vertical scale in each case: 0.4 units of $\mathrm{sM}(s)$ between each base line.

\section{CLUSTER FORMATION}

Electron diffraction patterns were recorded under a variety of conditions with various nozzle configurations, as indicated by Table 1 . All of the patterns obtained with pure butane could be quantitatively accounted for in terms of a sample of gas phase molecules exhibiting no aggregation, irrespective of the nozzle type. When carrier gas, either helium or neon, was added, conspicuous intensity features corresponding to clusters of butane molecules could be seen. In general, the largest cluster scattering occurred with low mole fractions of butane $(5 \%-25 \%)$ at the maximum pressure that our pumps could cope with for particular nozzles. Considerations of time available for aggregation before the expansions became too rarified for collisions to occur make it plausible to expect the strongest cluster signals to be associated with the largest nozzle sizes and with the nozzles giving the least divergent beams. Such tendencies, if present, are not clearly recognizable in the results tabulated in Table I. A more systematic investigation would be needed to establish trends.

In Table I, relative exposure times, referenced to be inversely proportional to the electron scattering power of the gas jet, are presented as a gauge of sample density. It should be noted that although thin plate nozzles were capable of producing large cluster signals, the density of the gas jet is at least 10-20 times less than for similar results achieved with tapered glass nozzles. The highly collimated jets produced by tapered nozzles reduce exposure times for an $R^{3}$ sector from several minutes to a few seconds, and scatter as strongly as gas jets in conventional gas electron diffraction studies.

One of the largest $n$-butane cluster intensities that we have yet seen is illustrated in Figs. 1-3 for plate 


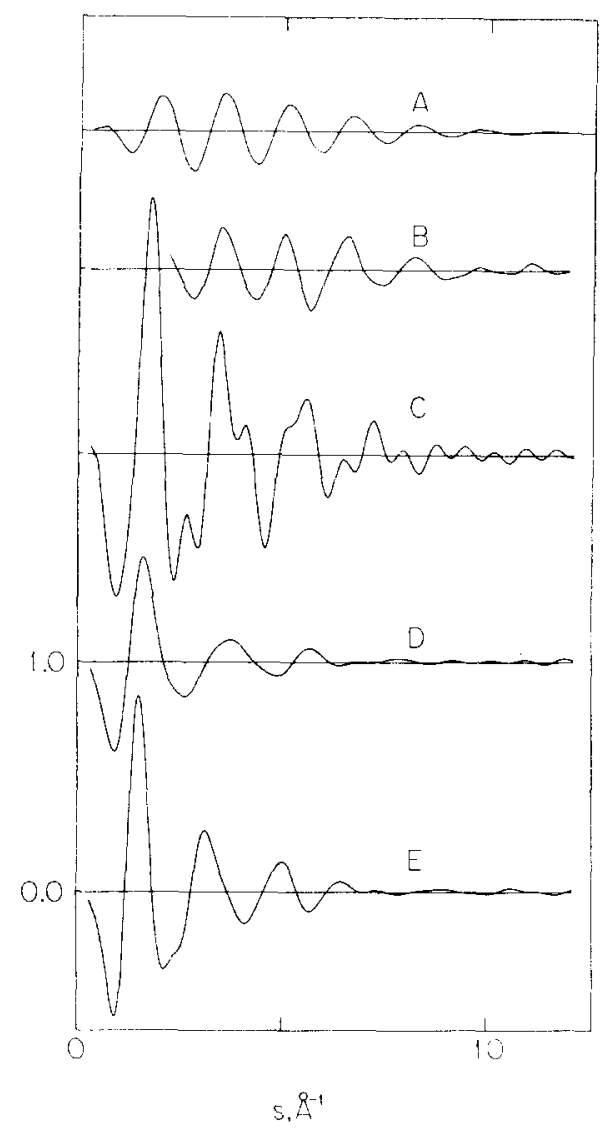

FIG. 3. $s M(s)$ curves. (a) Theoretical curve from $16 \mathrm{C} \cdots \mathrm{H}$ distances of $4 \AA \AA$ per monomeric unit with amplitude of vibration $l=0.25 \AA$. (b) Cluster scattering for plate 369 , as in Figs. 1 and 2. C-E. Theoretical intensities from $n$-butane liquid simulations, contain $\mathrm{C} \cdots \mathrm{C}$ contributions only: $\mathrm{C}, 76 \mathrm{~K}$ and $\mathrm{D}, 315 \mathrm{~K}$ from Weber, Ref. 4; E, at boiling point $272.5 \mathrm{~K}$, from Jorgensen et al. Ref. 1. Note that the high frequency ripples in $\mathrm{C}$ are a nonphysical artifact arising from our Fourier inversion of the limited $r$ range reported in Ref. 4.

369. As a convenient measure of the magnitude of such cluster intensities we include in Table I the height of the cluster signal around $s=5 \AA^{-1}$ on an $s M(s)$ plot. The same restrictions on interpretation of these numbers apply here as for benzene signals discussed in Ref. 6. This height depends not only on the average size of the clusters, but also on their structure and temperature, the fraction of free $n$-butane molecules remaining in the jet and on the model used to fit the monomer scattering.

Cluster scattering from plates such as 369 is dominated by components corresponding to interatomic distances of around $4 \AA$. These must be associated with nearest neighbor intermolecular C ...H distances since liquid simulations show characteristic intermolecular C ... C approaches to have typical values from $4 \cdot 4 \AA$, for $\mathrm{CH}_{3} \cdots \mathrm{CH}_{3}$, to around $5.3 \AA$ for $\mathrm{CH}_{3} \cdots \mathrm{CH}_{2}$ or $\mathrm{CH}_{2} \ldots \mathrm{CH}_{2} \cdot{ }^{1}$ Longer intermolecular distances also scatter electrons, of course, but the spectrum of distances becomes so dense and featureless that little detail is contributed to the pattern observed. Comparisons are made in Fig. 3 of the cluster intensity from plate 369 with a hypothetical $4 \AA \mathrm{C} \ldots \mathrm{H}$ term and with inten- sities calculated from published theoretical C . . C distributions. First we show calculations from molecular dynamics simulations of Weber at 76 and $315 \mathrm{~K} .{ }^{4}$ The simulation used 6-9 intermolecular potential energies and fully flexible, skeletal butane molecules. Also shown is a calculated intensity from a Monte Carlo computation by Jorgenson et al. ${ }^{1}$ at the boiling point. $\left(-0.5^{\circ} \mathrm{C}\right)$, based on Lennard-Jones, 6-12 intermolecular potentials and skeletal butane molecules with only internal rotation included. Unfortunately, because none of these studies provided $\mathrm{C} \ldots \mathrm{H}$ radial distributions, only $\mathrm{C} \cdots \mathrm{C}$ intensities could be calculated. In the case of electron diffraction but not $x$-ray diffraction the $\mathrm{C} \cdots \mathrm{H}$ terms contribute substantially to the scattering. Even though the requisite structural information on liquids is not yet available from theory, it is evident from the observed diffraction patterns that our clusters are essentially liquidlike. The size and rate of damping with $s$ of our largest experimental signals is in closer correspondence with the $76 \mathrm{~K}$ simulation of Weber than with the $315 \mathrm{~K}$ result. It is also likely for patterns similar to 369 that almost all of the $n$-butane molecules are involved in clusters. Temperatures below $200 \mathrm{~K}$ would be entirely consistent with our studies of benzene and $\mathrm{SF}_{6}$ clusters. $^{6,13}$

Although it should be possible to estimate an intermolecular vibrational temperature reasonably well, especially when more extensive scattering data become available for clusters, there is no direct indication of the conformational temperatures of the molecules within them. In fact, unless the effects of intermolecular cluster distances are carefully removed it is impossible to find the trans/gauche ratio of the $n$-butane monomers. This is because the long $(\mathrm{C} \cdots \mathrm{C})_{1,4}$ distance in trans $n$-butane that distinguishes this conformer from gauche is similar to the intermolecular $\mathrm{C} \cdots \mathrm{H}$ distances. If clusters are not allowed for, the apparent trans mole fraction increases to above $100 \%$ in our least-squares analysis. It can be seen in Fig. 2 that the theoretical $100 \%$ trans minus $68 \%$ trans signal that would appear with conformational cooling bears a resemblance at low $s$ to our cluster intensities. Reliable determination of conformational cooling requires careful tests for the presence of clusters. ${ }^{17}$ Though we may be confident of the shapes of large cluster signals such as 369 , weaker ones are much more affected by the assumed monomer conformation and by any systematic errors introduced by the monomer model. Two representative small cluster signals are shown in Fig. 2, from plates 314/5 (helium carrier) and plates $364 / 5$ (neon carrier).

\section{CONCLUDING REMARKS}

The present experiments show the feasibility of generating liquidlike microdroplets by nucleation in supersonic expansions. Prior systems studied by electron diffraction have led to predominantly solid clusters. In the case of $n$-butane strong condensation was not observed with the present micronozzles unless a large amount of carrier gas was present to lower temperatures sharply and to carry away the heat of condensation. Neon and helium were not strikingly different in their capacity to induce clustering. Nozzle design can alter 
the expansions markedly, but clustering was found for all types of nozzles, given sufficient carrier gas.

Electron diffraction patterns of intermolecular scattering from the microdrops exhibit a far greater signal-to-noise ratio than reported in prior studies of liquids by $x$-ray or neutron diffraction. This is partly due to counting statistics and partly to the low temperatures attained in supersonic flow. Our favorable cluster scattering signals should provide a major incentive for work in this area and may reveal useful information on the process of condensation as well as intermolecular interactions. In addition, the present supersonic studies afforded a view of the dynamics of gauche to trans conformational relaxation. This is described in the following paper.

\section{ACKNOWLEDGMENTS}

This research was supported by the National Science Foundation under grant number CHE-7926480. We thank E. J. Valente and T. Trull for their assistance with experimental work. A generous allocation of computer time was provided by the University of Michigan Computing Center.

${ }^{1}$ W. L. Jorgensen, R. C. Binning, and B. Bigot, J. Am. Chem. Soc. 103, 4393 (1981).

${ }^{2}$ W. L. Jorgensen, J. Am. Chem. Soc. 103, 4721 (1981). ${ }^{3}$ w. L. Jorgensen (unpublished).

'T. A. Weber, J. Chem. Phys. 69, 2347 (1978).

${ }^{5}$ See, for example, W. F. VanGusteren, H. J. C. Berendsen, and J. A. C. Rullmann, Mol. Phys. 44, 69 (1981); M. R. Pear and J. A. McCammon, J. Chem. Phys. 74, 6922 (1981); J. A. Montgomery, S. L. Holmgren, and D. Chandler, ibid. 73, 3688 (1980); R. M. Levy, M. Karplus, and J. A. McCammon, Chem. Phys. Lett. 65, 4 (1979).

${ }^{6}$ R. K. Heenan, E. J. Valente, and L. S. Bartell, J. Chem. Phys. 78, 243 (1983).

'A. H. Narten (personal communication).

${ }^{9}$ M. L. Cangeloni and V. Schettino, Mol. Cryst. Liq. Cryst. 31, 219 (1975).

'J. Devaure and J. Lascombe, Nouvo J. Chim. 3, 579 (1979).

${ }^{10}$ S. KInt, J. R. Scherer, and R. G. Snyder, J. Chem. Phys. 73, 2599 (1980).

${ }^{11}$ L. Colombo and G. Zerbi, J. Chem. Phys. 7S, 2013 (1980).

${ }^{12}$ D. A. C. Compton, S. Montero, and W. F. Murphy, J. Phys. Chem. 84, 3587 (1980).

${ }^{13}$ L. S. Bartell, K. R. Heenan, and M. Nagashima, J. Chem. Phys. 78, 236 (1983).

${ }^{14}$ See AIP document No. PAPS JCPSA-1265 for nine pages of tables containing experimental intengities, background functions, and intermolecular scattering for plates discussed in this work (papers III and IV). Order by PAPS number and journal reference from American Institute of Physics, Physics Auxiliary Publication Service, 335 E. 45 St. , New York, 10017. The price is $\$ 1.50$ for each microfiche (98 pages), or $\$ 5$ for photocopies of up to 30 pages and $\$ 0.15$ for each page over 30 pages. Airmail is additional. Make checks payable to American Institute of Physics.

${ }^{15}$ W. F. Bradford, S. Fitzwater, and L. S. Bartell, J. Mol. Struct. 38, 185 (1977).

${ }^{16} \mathrm{H}$. L. Sellers, L. Schafer, and R. A. Bonham, J. Mol. Struct. 49, 125 (1978).

${ }^{17}$ R. K. Heenan and L. S. Bartell, J. Chem. Phys, 78, 1270 (1983).

${ }^{18}$ L. S. Bartell, M. A. Kacner, and S. R. Goates, J. Chem. Phys. 75, 2730, 2736 (1981). 